\title{
Three Cases of Pierre Robin Sequence with Upper Airway Obstruction Relieved by Nasopharyngeal Airway Insertion
}

\author{
Min-su Oh ${ }^{1}$, Yu-Mi Park ${ }^{2}$, Young Hwa Jung ${ }^{2}$, Chang Won Choi ${ }^{2}$, Beyong Il Kim², and Ji-Won Kwon ${ }^{2}$ \\ ${ }^{1}$ Department of Pediatrics, Jeju National University Hospital, Jeju National University College of Medicine, Jeju, Korea \\ ${ }^{2}$ Department of Pediatrics, Seoul National University Bundang Hospital, Seoul National University College of Medicine, Seongnam, \\ Korea
}

\section{ABSTRACT}

Pierre Robin sequence (PRS), also called Robin sequence, is a congenital anomaly characterized by a triad of micrognathia, glossoptosis, and upper airway obstruction. Infants with PRS can present with varying degrees of respiratory difficulty secondary to upper airway obstruction. There has been no consensus for the treatment of upper airway obstruction in infants with PRS, but recent studies recommend attempting non-surgical interventions before surgical treatment. In this case report, we present 3 cases of infants diagnosed with PRS who showed persistent respiratory difficulties after birth. Before considering surgical intervention, insertion of a nasopharyngeal airway was attempted in these infants. Following this procedure, symptoms of upper airway obstruction were relieved, and all infants were discharged without surgical interventions; the nasopharyngeal airway was removed 1 to 2 months later. To date, no infant has shown signs of upper airway obstruction. Nasopharyngeal airway insertion is a highly effective and less invasive treatment option for infants with PRS. However, it is not widely known and used in Korea. Nasopharyngeal airway insertion can be preferentially considered before surgical intervention for upper airway obstruction in such infants.

Key Words: Pierre Robin syndrome; Upper airway obstruction; Nasopharyngeal airway

\section{INTRODUCTION}

Pierre Robin sequence (PRS) is a congenital anomaly characterized by a triad of micrognathia, glossoptosis, and upper airway obstruction, frequently accompanied by cleft palate ${ }^{1)}$. PRS is not considered a syndrome in itself but a sequence of disorders because one condition precipitates a sequence of events resulting in further signs and symptoms. Mandibular hypoplasia, with or without an underlying disorder occurring in the first trimester, leads
Received: 8 April 2019

Revised: 2 June 2019

Accepted: 19 June 2019

Correspondence to: Ji-Won Kwon

Department of Pediatrics, Seoul Na tional University Bundang Hospital, Seoul National University College of Medicine, 82 Gumi-ro 173beon-gil, Bundang-gu, Seongnam 13620, Korea Tel: +82-31-787-7296

Fax: +82-31-787-4054

E-mail:pedas@snubh.org https://orcid.org/0000-0002-5129-928X
Copyright(c)

By Korean Society of Neonatology.

All right reserved.

This is an Open-Access article distributed under the terms of the Creative Commons Attribution Non-Commercial License (http://creativecommons.org/licenses/ by-nc/4.0), which permits unrestricted non-commercial use, distribution, and reproduction in any medium, provided the original work is properly cited. 
to a reduction in the oropharyngeal space and a consequent upward and posterior displacement of the tongue, resulting in impaired palate closure and hypopharyngeal obstruction ${ }^{1,2)}$. Infants with PRS can present with varying degrees of upper airway obstruction with stridor, chest retraction, sleep apnea, cyanosis, and respiratory insufficiency ${ }^{3,4)}$. Additionally, $25 \%$ to $45 \%$ of infants with PRS present with feeding and swallowing difficulties, which may be associated with respiratory disorders ${ }^{4)}$.

The management of upper airway obstruction in infants with PRS includes non-surgical and surgical interventions. Non-surgical options include prone positioning, nasopharyngeal airway (NPA) insertion, continuous positive airway pressure (CPAP), and orthodontic apparatus. Surgical interventions include tongue-lip adhesion, mandibular distraction osteogenesis, and tracheostomy $^{1)}$. Usually, prone positioning is attempted before the other methods; however, there is a lack of consensus regarding the choice of treatment if prone positioning fails. Several studies have reported the effectiveness and safety of NPA insertion, and recent studies have shown that the high success rate of NPA insertion is similar to that of surgical interventions ${ }^{1}$. Therefore, NPA insertion can be considered as a primary treatment option prior to performing surgery in infants with PRS (Figure 1) $)^{5}$. In spite of this, NPA insertion has not been widely used as routine airway management for PRS in Korea. We report three cases of infants with PRS who presented with upper airway obstruction which was relieved by NPA insertion.

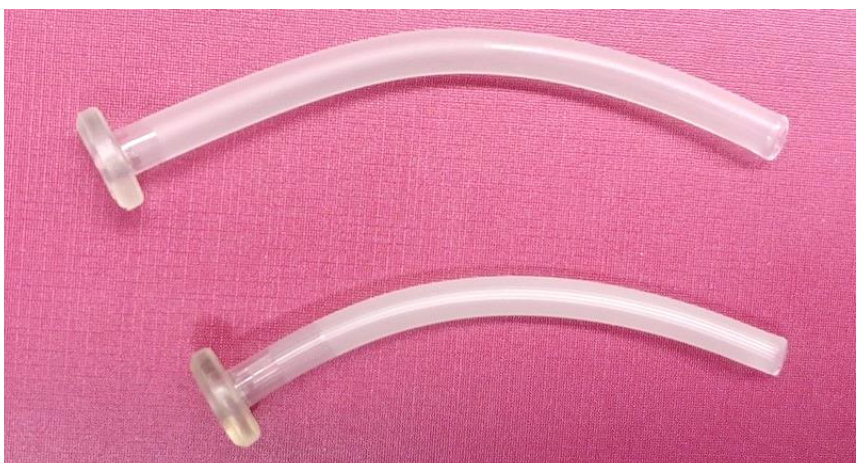

Figure 1. Nasopharyngeal airways. The length of the tube can be selected by measuring the distance between the nares and the earlobe, which should be confirmed radiographically on a lateral neck radiograph to check if the tube is appropriately placed just above the epiglottis ${ }^{11)}$.

\section{CASE REPORTS}

\section{Case 1}

A 68-day-old male infant was admitted to our hospital with signs of recurrent upper airway obstruction. He was born at the other hospital at a gestational age of $40^{+3}$ weeks with a birth weight of $3.24 \mathrm{~kg}$ (10th to 50th percentile). Immediately after birth, he showed respiratory difficulty with desaturation and chest retraction. He was admitted to the neonatal intensive care unit (NICU) and diagnosed with PRS, based on micrognathia, glossoptosis, and cleft palate. Although a high flow nasal cannula (HFNC) was applied, he showed intermittent desaturation secondary to upper airway obstruction. Gastrostomy was performed on the 38th day after birth owing to a difficulty in oral feeding. Brain ultrasonography and echocardiography were unremarkable except for patent foramen ovale.

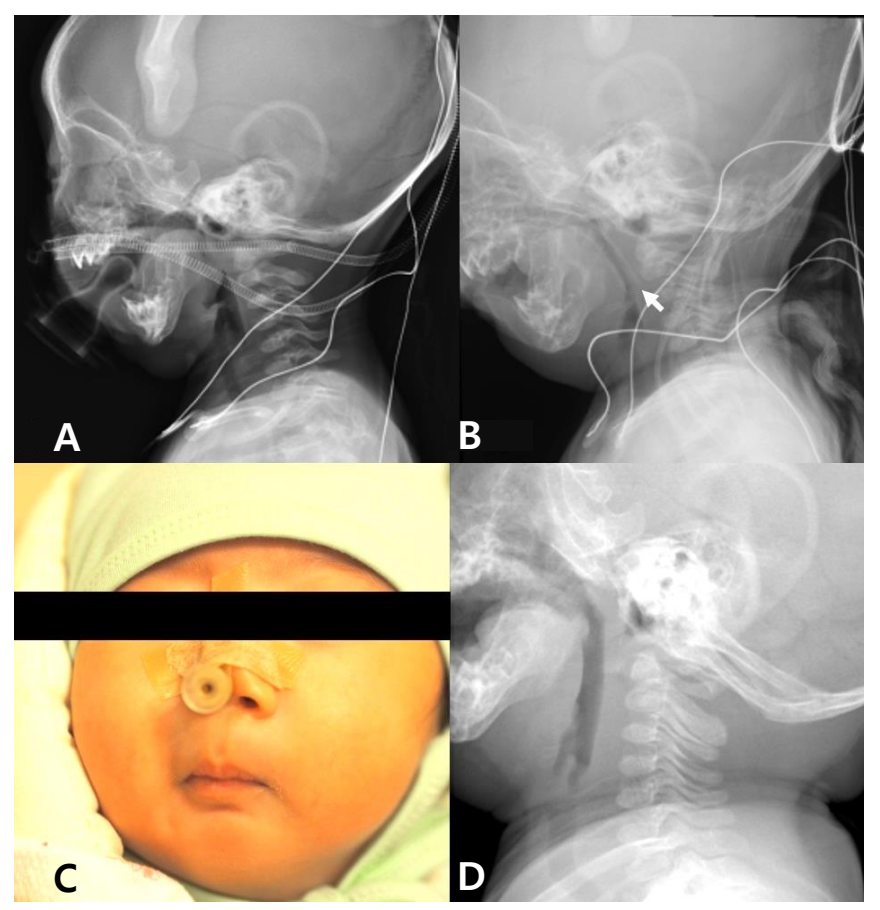

Figure 2. Cephalometry radiographs and photograph in case 1. (A) Representative case of Pierre Robin sequence in a 2month-old infant showing micrognathia and glossoptosis with features of upper airway obstruction. (B) Image showing a nasopharyngeal airway inserted into the larynx, thereby moving the tongue forward and bypassing the tongue base. The white arrow indicating the tip of nasopharyngeal airway placed above the epiglottis. (C) Image of the same infant maintaining a nasopharyngeal airway after discharge (D) Image of the same infant at 4 months of age showing that following mandibular growth, upper airway patency is maintained without the insertion of a nasopharyngeal airway. 
He was referred to our hospital for further management, including a tracheostomy, and weighed $4.24 \mathrm{~kg}$ ( $<3 \mathrm{rd}$ percentile) upon admission. Pulse oximetry monitoring showed intermittent desaturation despite HFNC (flow rate $7 \mathrm{~L} / \mathrm{min}, \mathrm{FiO}_{2}$ 0.30). Cephalometry radiographs showed hypopharyngeal obstruction secondary to glossoptosis (Figure 2). A 3.5 Fr NPA (Rusch ${ }^{\circledR}$, Teleflex, Morrisville, NC, USA) insertion was performed on hospital day 2, after which symptoms of upper airway obstruction were relieved, and HFNC was discontinued. His parents were educated regarding management of the NPA, and he was discharged with the NPA on hospital day 17. The NPA was successfully removed 12 days after discharge and the gastrostomy tube was also removed when he was able to feed orally. To date, he has not experienced recurrent breathing difficulty or signs of upper airway obstruction. He weighs $11.9 \mathrm{~kg}$ (25th to 50 th percentile) at 24 months of age.

\section{Case 2}

An 86-day-old male infant was transferred to our hospital after several failed attempts of ventilator weaning. He was born at a gestational age of $33^{+2}$ weeks with a birth weight of $2.12 \mathrm{~kg}$ (50th percentile). After birth, he was admitted to the NICU secondary to respiratory difficulty and desaturation where he was diagnosed with PRS based on micrognathia, glossoptosis, and cleft palate. He had no anomalies on brain ultrasonography and echocardiography, and developed recurrent episodes of upper airway obstruction requiring noninvasive positive pressure ventilation support; however, multiple weaning attempts failed. Repeated regurgitation and aspiration pneumonia necessitated feeding via a gavage tube. Tongue-lip adhesion was recommended before transfer to our hospital.

Upon admission, the patient weighed $3.87 \mathrm{~kg}$ ( $<3 \mathrm{rd}$ percentile). Bronchoscopic examination showed posterior displacement of the tongue base with retrognathia and mild laryngomalacia. We inserted a 3.5 Fr NPA and were able to wean him off the ventilator. No significant upper airway obstruction was observed thereafter. The NPA was successfully removed on the 149th day of birth. Regurgitation also improved, and at present oral feeding has been established. He weighs $8.4 \mathrm{~kg}$ (10th to 25th percentile) at 10.5 months of age.

\section{Case 3}

A female infant born at a gestational age of $36^{+6}$ weeks with a birth weight of $2.1 \mathrm{~kg}$ (3rd to10th percentile) presented with chest retraction and tachypnea 8 hours after birth. She had a cleft palate and feeding difficulty and was therefore transferred to our NICU for further management 1 day after birth. She was diagnosed with PRS based on micrognathia and glossoptosis and did not have anomalies on brain ultrasonography and echocardiography. Although she did not require respiratory support, mild stridor and intermittent desaturation were observed in the supine position. Despite the use of a special bottle for cleft palate, she developed recurrent episodes of oxygen desaturation during feeding with frequent episodes of upper airway obstruction. Despite a gradual increase in feeding volume, weight gain was poor, and she weighed $2.19 \mathrm{~kg}$ at 27 days of age. We inserted a 3.0 Fr NPA as a primary treatment for desaturation during feeding, and after NPA insertion, she showed no desaturation during oral feeding or in the supine position. Her weight increased to 2.68 $\mathrm{kg}$ at 44 days after birth, and she was discharged with the NPA. The NPA was removed on the 86th day of birth. To date, she has shown adequate feeding and weight gain without signs of upper airway obstruction. She weighs $7.2 \mathrm{~kg}$ (5th tol0th percentile) at 11 months of age.

This study was approved by the Institutional Review Board of Seoul National University Bundang Hospital, which was granted a waiver of informed consent (approval no. B-1907/552-701).

\section{DISCUSSION}

This is the first case report describing the use of NPA to treat infants with PRS in Korea. These infants presented with recurrent upper airway obstruction and desaturation, and surgical interventions such as tongue-lip adhesion or tracheostomy procedures had been considered before NPA insertion. Symptoms of airway obstruction were relieved after NPA insertion, and the infants were discharged with the NPA. To date, these infants have developed no recurrence of upper airway obstruction and have been fed orally after NPA removal.

PRS was first described by Pierre Robin in $1923^{6}$; however, there is a lack of consensus regarding optimal treatment modalities for this condition. Although no randomized studies have compared the available treatments, recent studies recommend a trial of non-surgical intervention preferentially in infants who fail prone positioning. Results of 14 studies performed across 10 different countries between 1962 and 2011 have shown that of the 1,425 infants with PRS who were investigated, $68 \%$ were treated suc- 
cessfully with non-surgical intervention such as prone positioning, NPA insertion, orthodontic apparatus, and CPAP ${ }^{1)}$. A 10year retrospective study performed in 2017 reported that $76 \%$ of the 63 infants investigated were successfully managed by prone positioning and NPA insertion ${ }^{7)}$. A systematic review that summarized the results of 48 studies investigating infants with PRS determined that the success rates of NPA insertion, orthodontic apparatus, non-invasive respiratory support such as CPAP, tongue-lip adhesion, subperiosteal release of the floor of the mouth, mandibular traction, and tracheostomy were $67 \%$ to 100 $\%(n=126), 90 \%(n=11), 100 \%(n=12), 70 \%$ to $96 \%(n=277), 50 \%$ to $84 \%(n=47), 100 \%(n=133)$, and $100 \%(n=25)$, respectively ${ }^{3)}$. Because of similar success rates between non-surgical and surgical interventions, they recommended a trial of non-surgical intervention preferentially in the event of failure of prone positioning .

In Korea, several studies have reported surgical interventions for infants with PRS. The first case of PRS in Korea was reported in 1969, which described surgical and conservative treatment ${ }^{8)}$. The primary surgical success rate associated with tongue-lip adhesion was $66.7 \%$ among eight cases of PRS reported between 1992 and $2011^{9)}$. Another case reported in 2008 described successful mandibular distraction osteogenesis in a 7-month-old infant with PRS in whom symptoms of upper airway obstruction persisted after undergoing tongue-lip adhesion ${ }^{10)}$.

Previous studies have reported that a number of infants with PRS show an increased mandibular growth rate and relatively slower growth of the tongue. This results in spontaneous improvement of airway obstruction during the first year of life ${ }^{1)}$. Therefore, non-surgical interventions can be a reasonable therapeutic option while awaiting mandibular growth. Prone positioning can be easily attempted and has shown a $40 \%$ to $70 \%$ success rate $^{5)}$. However, continuous monitoring of breathing is required, and this strategy usually fails in infants with severe upper airway obstruction.
Although there are no studies comparing each non-surgical intervention, the successful use of NPA insertion has been more frequently reported than other interventions when prone position failed $^{1,7)}$. NPA insertion can be tried more easily and immediately without any apparatus- or mask-related discomfort. The NPA is a flexible tube inserted into the nostril with the distal end of the tube positioned in the larynx, just above the epiglottis $(\text { Figure } 1)^{11)}$. This device allows infants to breathe through it, by moving their tongue forward and bypassing the tongue base ${ }^{5)}$. The NPA is generally used in the management of congenital maxillofacial abnormalities, syndromic craniosynostosis, midfacial hypoplasia, upper airway post-trauma or surgery, and emergency upper airway obstruction ${ }^{12)}$.

In infants with PRS, NPA insertion is a useful alternative even in cases of moderate-to-severe airway obstruction in infants not responding to prone positioning ${ }^{5}$. Because NPA insertion is a simple procedure associated with low morbidity, infants with PRS undergoing NPA insertion can be safely managed at home after parental education on NPA care. An 11-year retrospective study performed in 2012 reported that 63 of 104 infants (60.6\%) with PRS were managed with NPA ${ }^{13)}$. In a recent study of 107 infants with PRS with severe respiratory difficulty who improved following NPA insertion, the mean duration of NPA use was 57 days (range, 1 to 173), and the mean length of hospitalization was 18 days (range, 2 to 57$)^{14)}$. No infant developed complications associated with NPA use, and the NPA was successfully removed without the need for tracheostomy in all cases. In the cases reported in our study, the NPA was maintained for a mean of 50.3 days (range, 28 to 63 ) and removed when the infants were 86 to 149 days old (Table 1 ).

Other causes of airway obstruction should be excluded in infants who remain symptomatic even after NPA insertion. A few studies have reported that invasive surgical interventions were needed in infants with syndromic PRS ${ }^{3)}$. Syndromic PRS

Table 1. Summary of Cases

\begin{tabular}{|c|c|c|c|}
\hline & Case 1 & Case 2 & Case 3 \\
\hline Reason for NPA insertion & Weaning failure of HFNC & Weaning failure of NIPPV & Feeding desaturation \\
\hline Age of insertion & 69 days & 86 days & 26 days \\
\hline Duration of NPA insertion & 28 days & 63 days & 60 days \\
\hline Birth weight & $3.24 \mathrm{~kg}(10-50 \mathrm{th})$ & $2.12 \mathrm{~kg}$ (50th) & $2.1 \mathrm{~kg}$ (3-10th) \\
\hline Weight before NPA insertion & $4.24 \mathrm{~kg}(<3 \mathrm{rd})$ & $3.87 \mathrm{~kg}(<3 \mathrm{rd})$ & $2.19 \mathrm{~kg}(<3 \mathrm{rd})$ \\
\hline Weight at present & $11.9 \mathrm{~kg}$ (25-50th at 24 months) & $8.4 \mathrm{~kg}$ (10-25th at 10 months $)$ & $7.2 \mathrm{~kg}$ (5-10th at 11 months) \\
\hline
\end{tabular}

Abbreviations: NPA, nasopharyngeal airway; HFNC, high flow nasal cannula; NIPPV, noninvasive positive pressure ventilation. 
may be associated with multilevel airway obstruction, as well as cardiac and neurological anomalies. Additionally, infants with syndromic PRS show lesser natural mandibular growth than infants with isolated PRS, resulting in lesser improvement of airway obstruction $^{3)}$. Syndromic PRS is observed in $27 \%$ to $70 \%$ of all infants with PRS and shows poorer outcomes than those observed in infants with isolated PRS. A study reported by $\mathrm{Li}$ et al. ${ }^{7)}$ determined that syndromic PRS was diagnosed in $47 \%$ (seven of 15) of patients in the surgical group versus $21 \%$ (10 of 48) of patients in the non-surgical group.

Notably, surgical interventions such as tongue-lip adhesion and mandibular distraction osteogenesis show higher failure rates in infants with syndromic PRS. Tracheostomy can be performed when all other treatments fail, particularly in infants with PRS with multilevel airway obstruction ${ }^{15)}$. However, tracheostomy is associated with higher risks of serious complications than other treatment modalities; therefore, the risk-benefit ratio should be carefully considered prior to selecting this approach. All infants reported in our study had isolated PRS without other anomalies and showed no developmental disorder in the outpatient followup. For serious airway obstruction in syndromic PRS, surgical interventions might be considered first hand.

In conclusion, NPA insertion is a highly effective and less invasive treatment option in infants with PRS presenting with moderate to severe upper airway obstruction. NPA insertion can be first tried before surgical intervention for the treatment of upper airway obstruction in infants with PRS.

\section{CONFLICT OF INTEREST}

No potential conflict of interest relevant to this article was reported.

\section{REFERENCES}

1. Cote A, Fanous A, Almajed A, Lacroix Y. Pierre Robin sequence: review of diagnostic and treatment challenges. Int J Pediatr Otorhinolaryngol 2015;79:451-64.

2. Rathe M, Rayyan M, Schoenaers J, Dormaar JT, Breuls M, Verdonck A, et al. Pierre Robin sequence: management of re- spiratory and feeding complications during the first year of life in a tertiary referral centre. Int J Pediatr Otorhinolaryngol 2015; 79:1206-12.

3. van Lieshout MJ, Joosten KF, Mathijssen IM, Koudstaal MJ, Wolvius EB, van der Schroeff MP. Non-surgical and surgical interventions for airway obstruction in children with Robin sequence. J Craniomaxillofac Surg 2016;44:1871-9.

4. Cladis F, Kumar A, Grunwaldt L, Otteson T, Ford M, Losee JE. Pierre Robin sequence: a perioperative review. Anesth Analg 2014;119:400-12.

5. Gomez OJ, Baron OI, Penarredonda ML. Pierre Robin sequence: an evidence-based treatment proposal. J Craniofac Surg 2018; 29:332-8.

6. Robin P. La chute de la base de la langue considérée comme une nouvelle cause de gêne dans la respiration naso-pharyngienne. Bull Acad Med 1923;89:37-41.

7. Li WY, Poon A, Courtemanche D, Verchere C, Robertson S, Bucevska M, et al. Airway management in Pierre Robin sequence: the Vancouver classification. Plast Surg (Oakv) 2017;25:14-20.

8. Chang IK, Rhee YW, Lew JD. Pierre Robin syndrome: case report. J Korean Surg Soc 1969;11:413-8.

9. Lee JW, Park BY. Tongue-lip adhesion using an alveolar protector appliance for management of Pierre Robin sequence. J Korean Soc Plast Reconstr Surg 2011;38:547-51.

10. Ryu SY, Kwon JK, Kim SK. Mandibular distraction osteogenesis in an infant with Pierre Robin sequence: report of a case. J Korean Oral Maxillofac Surg 2008;34:460-7.

11. Roberts $\mathrm{K}$, Whalley $\mathrm{H}$, Bleetman $\mathrm{A}$. The nasopharyngeal airway: dispelling myths and establishing the facts. Emerg Med J 2005; 22:394-6.

12. Tweedie DJ, Skilbeck CJ, Lloyd-Thomas AR, Albert DM. The nasopharyngeal prong airway: an effective post-operative adjunct after adenotonsillectomy for obstructive sleep apnoea in children. Int J Pediatr Otorhinolaryngol 2007;71:563-9.

13. Abel F, Bajaj Y, Wyatt M, Wallis C. The successful use of the nasopharyngeal airway in Pierre Robin sequence: an 11-year experience. Arch Dis Child 2012;97:331-4.

14. Drago Marquezini Salmen IC, Lazarini Marques I. In situ and home care nasopharyngeal intubation improves respiratory condition and prevents surgical procedures in early infancy of severe cases of robin sequence. Biomed Res Int 2015;2015: 608905.

15. Evans KN, Sie KC, Hopper RA, Glass RP, Hing AV, Cunningham ML. Robin sequence: from diagnosis to development of an effective management plan. Pediatrics 2011;127:936-48. 